\title{
Embedding Learning Through Multilingual Concept Induction
}

\author{
Philipp Dufter ${ }^{1}$, Mengjie Zhao ${ }^{2}$, Martin Schmitt ${ }^{1}$, Alexander Fraser ${ }^{1}$, Hinrich Schütze ${ }^{1}$ \\ ${ }^{1}$ Center for Information and Language Processing (CIS) LMU Munich, Germany \\ ${ }^{2}$ École Polytechnique Fédérale de Lausanne, Switzerland \\ \{philipp, martin, fraser\}@cis.lmu.de, mengjie.zhaodepfl.ch
}

\begin{abstract}
We present a new method for estimating vector space representations of words: embedding learning by concept induction. We test this method on a highly parallel corpus and learn semantic representations of words in 1259 different languages in a single common space. An extensive experimental evaluation on crosslingual word similarity and sentiment analysis indicates that concept-based multilingual embedding learning performs better than previous approaches.
\end{abstract}

\section{Introduction}

Vector space representations of words are widely used because they improve performance on monolingual tasks. This success has generated interest in multilingual embeddings, shared representation of words across languages (Klementiev et al., 2012). Such embeddings can be beneficial in machine translation in sparse data settings because multilingual embeddings provide meaning representations of source and target in the same space. Similarly, in transfer learning, models trained in one language on multilingual embeddings can be deployed in other languages (Zeman and Resnik, 2008; McDonald et al., 2011; Tsvetkov et al., 2014). Automatically learned embeddings have the added advantage of requiring fewer resources for training (Klementiev et al., 2012; Hermann and Blunsom, 2014b; Guo et al., 2016). Thus, massively multilingual word embeddings (i.e., covering 100 s or 1000 s of languages) are likely to be important in NLP.

The basic information many embedding learners use is word-context information; e.g., the embedding of a word is optimized to predict a representation of its context. We instead learn em-

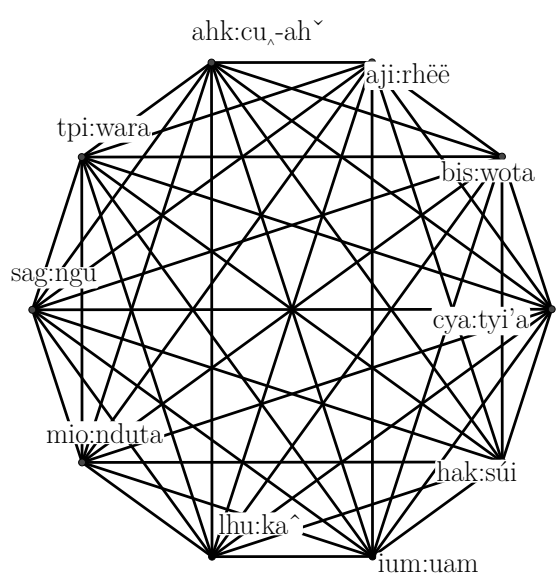

Figure 1: Example of a CLIQUE concept: "water"

beddings from word-concept information. As a first approximation, a concept is a set of semantically similar words. Figure 1 shows an example concept and also indicates one way we learn concepts: we interpret cliques in the dictionary graph as concepts. The nodes of the dictionary graph are words, its edges connect words that are translations of each other. A dictionary node has the form prefix:word, e.g., "tpi:wara" (upper left node in the figure). The prefix is the ISO 639-3 code of the language; tpi is Tok Pisin.

Our method takes a parallel corpus as input and induces a dictionary graph from the parallel corpus. Concepts and word-concept pairs are then induced from the dictionary graph. Finally, embeddings are learned from word-concept pairs.

A key application of multilingual embeddings is transfer learning. Transfer learning is mainly of interest if the target is resource-poor. We therefore select as our dataset 1664 translations in 1259 languages of the New Testament from PBC, the Parallel Bible Corpus. Since "translation" is an ambiguous word, we will from now on refer to the 1664 translations as "editions". PBC is aligned 
Table 1: Instances of verse 11018034. This multi-sentence verse is an example of verse misalignment.

on the verse level; most verses consist of a single sentence, but some contain several (see Table 1). PBC is a good model for resource-poverty; e.g., the training set (see below) of KJV contains fewer than 150,000 tokens in 6458 verses.

We evaluate multilingual embeddings on two tasks, roundtrip translation (RT) and sentiment analysis. RT on the word level is - to our knowledge - a novel evaluation method: a query word $w$ of language $L_{1}$ is translated to its closest (with respect to embedding similarity) neighbor $v$ in $L_{2}$ and then backtranslated to its closest neighbor $w^{\prime}$ in $L_{1}$. RT is successful if $w=w^{\prime}$. There are well-known concerns about RT when it is used in the context of machine translation. A successful roundtrip translation does not necessarily imply that $v$ is of high quality and it is not possible to decide whether an error occurred in the forward or backward translations. Despite these concerns about RT on the sentence level, we show that RT on the word level is a difficult task and an effective measure of embedding quality.

Contributions. (i) We introduce a new embedding learning method, multilingual embedding learning through concept induction. (ii) We show that this new concept-based method outperforms previous approaches to multilingual embeddings. (iii) We propose both word-level and characterlevel dictionary induction methods and present evidence that concepts induced from word-level dictionaries are better for easily tokenizable languages and concepts induced from character-level dictionaries are better for difficult-to-tokenize languages. (iv) We evaluate our methods on a corpus of 1664 editions in 1259 languages. To the best of our knowledge, this is the first detailed evaluation, involving challenging tasks like word translation and crosslingual sentiment analysis, that has been done on such a large number of languages.

\section{Methods}

\subsection{Pivot languages}

Most of our methods are based on bilingual dictionary graphs. With 1664 editions, it is computationally expensive to consider all editions si- multaneously (more than $10^{6}$ dictionaries). Thus we split the set of editions in 10 pivot and 1654 remaining editions, and do not compute nor use dictionaries within the 1654 editions. We refer to the ten pivot editions as pivot languages and give them a distinct role in concept induction. We refer to all editions (including pivot editions) as target editions. Thus, a pivot edition has two roles: as a pivot language and as a target edition.

We select the pivot languages based on their sparseness. Sparseness is a challenge in NLP. In the case of embeddings, it is hard to learn a high-quality embedding for any infrequent word. Many of the world's languages (including many PBC languages) exhibit a high degree of sparseness. But some languages suffer comparatively little from sparseness when simple preprocessing like downcasing and splitting on whitespace is employed.

A simple measure of sparseness that affects embedding learning is the number of types. Fewer types is better since their average frequency will be higher. Table 2 shows the ten languages in PBC that have the smallest number of types in 5000 randomly selected verses. We randomly sample 5000 verses per edition and compare the number of types based on this selection because most editions do not contain a few of the selected 6458 verses.

\subsection{Character-level modeling (CHAR)}

We will see that tokenization-based models have poor performance on a subset of the 1259 languages. To overcome tokenization problems, we represent a verse of length $m$ bytes, as a sequence of $m-(n-1)+2$ overlapping byte $n$-grams. In this paper, " $n$-gram" always refers to "byte $n$ gram". We pad the verse with initial and final space, resulting in two additional $n$-grams (hence " +2 "). This representation is in the spirit of earlier byte-level processing, e.g., (Gillick et al., 2016). There are several motivations for this. (i) We can take advantage of byte-level generalizations. (ii) This is robust if there is noise in the byte encoding. (iii) Characters have different properties in different languages and encodings, e.g., English 


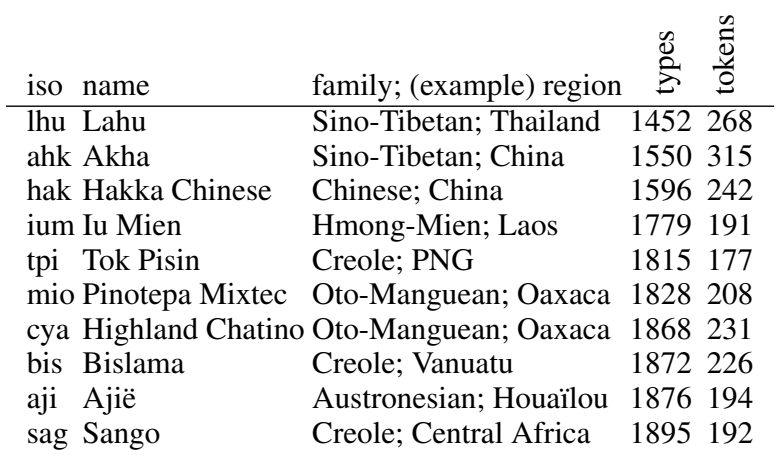

Table 2: Our ten pivot languages, the languages in PBC with the lowest number of types. Tokens in 1000s. Tok Pisin and Bislama are English-based and Sango is a Ngbandi-based creole. $\mathrm{PNG}=$ Papua New Guinea

UTF-8 has properties different from Chinese UTF8 . Thus, universal language processing is easier to design on the byte level.

We refer to this ngram representation as CHAR and to standard tokenization as WORD.

\subsection{Dictionary induction}

Alignment-based dictionary. We use fastalign (Dyer et al., 2013) to compute word alignments and use GDFA for symmetrization. All alignment edges that occurred at least twice are added to the dictionary graph. Initial experiments indicated that alignment-based dictionaries have poor quality for CHAR, probably due to the fact that overlapping ngram representations of sentences have properties quite different from the tokenized sentences that aligners are optimized for. Thus we use this dictionary induction method only for WORD and developed the following alternative for CHAR.

Correlation-based dictionary $\left(\chi^{2}\right) . \quad \chi^{2}$ is a greedy algorithm, shown in Figure 2, that selects, in each iteration, the pair of units that has the highest $\chi^{2}$ score for cooccurrence in verses. Each selected pair is added to the dictionary and removed from the corpus. Low-frequency units are selected first and high-frequency units last; this prevents errors due to spurious association of highfrequency units with low-frequency units. We perform $d_{\max }=5$ passes; in each pass, the maximum degree of a dictionary node is $1 \leq d \leq d_{\max }$. So if the node has reached degree $d$, it is ineligible for additional edges during this pass. Again, this avoids errors due to spurious association of highfrequency units that already participate in many

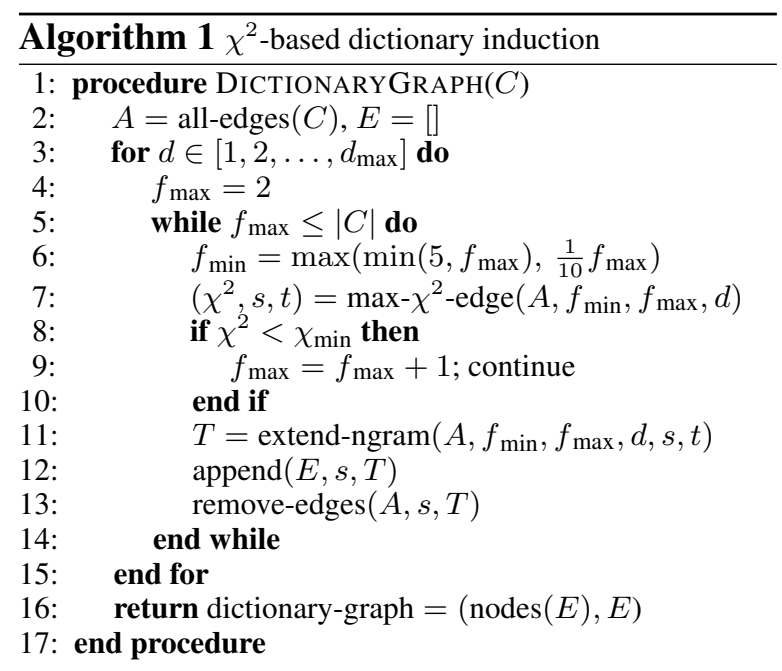

Figure 2: $\chi^{2}$-based dictionary induction. $C$ is a sentence-aligned corpus. $A$ is initialized to contain all edges, i.e., the fully connected bipartite graph for each parallel verse. $E$ collects the selected dictionary edges. $d$ is the edge degree: in each pass through the loop only edges are considered whose participating units have a degree less than $d . f_{\max }$ is the maximum frequency during this pass. $|C|$ is the number of sentences in the corpus. extend-ngram extends a target ngram to left / right; e.g., if $s=$ "jisas" is aligned with ngram $t=$ "Jesu" in English, then "esus" is added to T. $t$ is always a member of $T$. remove-edges removes edges in $A$ between $s$ and a member of $T$.

edges with low-frequency units. Recall that this method is only applied for CHAR.

Intra-pivot dictionary. We assume that pivot languages are easily tokenizable. Thus we only consider alignment-based dictionaries (in total 45) within the set of pivot languages.

Pivot-to-target dictionary. We compute an alignment-based and a $\chi^{2}$-based dictionary between each pivot language and each target edition, yielding a total of $10^{*} 1664$ dictionaries per dictionary type. (Note that this implies that, for $\chi^{2}$, the WORD version of the pivot language is aligned with its CHAR version.)

\subsection{Concepts}

A concept is defined as a set of units that has two subsets: (i) a defining set of words from the ten pivot languages and (ii) a set of target units (words or $n$-grams) that are linked, via dictionary edges, 


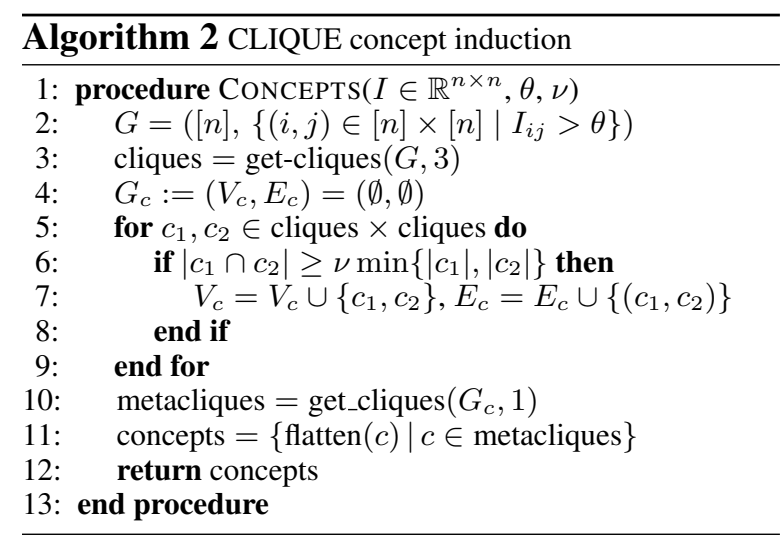

Figure 3: CLIQUE concept induction. I is a normalized adjacency matrix of a dictionary graph (i.e., relative frequency of alignment edges with respect to possible alignment edges). get-cliques $(G, n)$ returns all cliques in $G$ of size greater or equal to $n$. flatten $(A)$ flattens a set of sets. $[n]$ denotes $\{1,2, \ldots, n\} . \theta=0.4, \nu=0.6$.

to the pivot subset. We selected the ten "easiest" of the 1664 editions as pivot languages. Our premise is that semantic information is encoded in a simply accessible form in the pivot languages and so they should offer a good basis for learning concepts.

We induce concepts from the dictionary graph, a multipartite graph consisting of ten pivot language node/word sets and all target edition node/unit sets (where units are words or $n$-grams). Edges either connect pivot nodes with other pivot nodes or pivot nodes with target units.

\subsubsection{CLIQUE concept induction}

If concepts corresponded to each other in the overtly coding pivot languages, if words were not ambiguous and if alignments were perfect, then concepts would be cliques in the pivot part of the dictionary graph. These conditions are too strict for natural languages, so we relax them in our CLIQUE concept induction algorithm (Figure 3). The algorithm identifies maximal multilingual cliques (size $\geq 3$ ) within the dictionary graph of the pivot languages and then merges two cliques if they share enough common words. The merging lets us identify clique-based concepts even if, e.g., a dictionary edge between two words is missing. It also accommodates the situation where more than one word of a pivot language should be part of a concept. The merging step can also be interpreted as metaconcept induction.

Once we have identified the cliques, we project
$N(t)=\{$ bis:Jorim, ium:yo-lim, sag:Yorim, tpi:Jorim $\}$

$t \in T=\{$ ac0: Yorim, atg 0 : iJorimu, bav0: Jorim, bom0: Yorim, dik0:Jorim, dtp0:Yorim, duo0:Jorim, eng1:Jorim, engb:Jorim, fij2:Lorima, fij3:Jorima, gor0:Yorim, hvn0:Yorim, ibo0:Jorim, irio:Jorri, kmr0:Yorîm, ksd0:Iorim, kwd0:Jorim, lia0:Yorimi, loz0:Jorimi, mbd0:Hurim, mfho:Yorim, mino:Yorim, mrw0:Yorim, mse0: Jorimma, naq0:Jorimmi, smo1:Iorimo, srn1:Yorim, tsn2:Jorime, yor2:Jórímù

Figure 4: Target neighborhood concept example: $N(t) \cup T . N(t)$ is the target neighborhood for each of the target words in $T$.

them to the target editions: a target-unit is added to a clique if it is connected to a proportion $\nu=0.6$ of its member words (to allow for missing edges). This identifies around 150k clique concepts that cover around $8 \mathrm{k}$ of the total vocabulary of $24 \mathrm{k}$ English words (WORD).

As an alternative to cliques, Ammar et al. (2016) use connected components (CCs). The reachability relation (induced by $\mathrm{CC}$ ) is the transitive closure of the edge relation. This results in semantically unrelated words being in the same concept for very low levels of noise. In contrast, cliques are more "strict": only node subsets are considered whose corresponding edge relation is already transitive (or almost so for $\nu=0.6$ ). Transitivity across languages often does not hold in alignments or dictionaries; see, e.g., Simard (1999). This is why we only consider cliques (which reflect already existent transitivity) rather than CCs, which impose transitivity where it does not hold naturally.

\subsection{2 $N(t)$ (target neighborhood) concept induction}

Let $N(t)$ be the neighborhood of target node $t$ in the multipartite dictionary graph, i.e., the set of pivot words that are linked to $t$. We refer to $N(t)$ as target neighborhood. Figure 4 shows an example of such a target neighborhood, the set $N(t)$ consisting of four words. ${ }^{1}$ A target neighborhood concept consists of a set $T$ of pivot words and all target words $t$ for which $T=N(t)$ holds.

Motivation. Suppose $N(t)=N(u)$ for target nodes $t$ and $u$ from two different languages and $|N(t)|$ covers several pivot languages, e.g., $|N(t)|=|N(u)|=4$ as in the figure. Again, if units closely corresponded to concepts, if there were no ambiguity, if the dictionary were perfect,

\footnotetext{
${ }^{1}$ We use numbers and lowercase letters at the fourth position of the prefix to distinguish different editions in the same language, e.g., "0", "3" and "e" in "ace0", "fij3", "enge".
} 
then we could safely conclude that the meanings of $t$ and $u$ are similar; if the meanings of $t$ and $u$ were unrelated, it is unlikely that they would be aligned to the exact same words in four different languages. In reality, there is no exact meaningform correspondence, there is ambiguity and the dictionary is not perfect. Still, we will see below that defining concepts as target neighborhoods works well.

\subsubsection{Filtering target neighborhood concepts}

In contrast to CLIQUE, we do not put any constraint on the pivot-to-pivot connections within target neighborhoods; e.g., in Figure 4, we do not require that "bis:Jorim" and "sag:Yorim" are connected by an edge. We evaluate three postfiltering steps of target neighborhoods to increase their quality: restricting target neighborhoods to those that are cliques in $N(t)$-CLIQUE; to those that are connected components in $N(t)-\mathbf{C C}$; and to those of size two that are valid edges in the dictionary in $N(t)$-EDGE. For $N(t)$-EDGE, we found that taking all edges performs well, so we also consider edges that are proper subsets of target neighborhoods.

\subsection{Embedding learning}

We adopt the framework of embedding learning algorithms that define contexts and then sample pairs of an input word (more generally, an input unit) and a context word (more generally, a context unit) from each context. The only difference is that our contexts are concepts. For simplicity, we use word2vec (Mikolov et al., 2013a) as the implementation of this model. ${ }^{2}$

\subsection{Baselines}

Baselines for multilingual embedding learning. One baseline is inspired by (Vulić and Moens, 2015). We consider words of one aligned verse in the pivot languages and one target language as a bag of words (BOW) and consider this bag as a context. $^{3}$

Levy et al. (2017) show that sentence ID features (interpretable as an abstract representation of the word's context) are effective. We use a corpus with lines consisting of pairs of an identifier of a

\footnotetext{
${ }^{2}$ We use code.google.com/archive/p/word2vec

${ }^{3}$ The actual implementation slightly differs to avoid very long lines. It does only consider two pivot languages at a time, but writes each verse multiple times.
}

verse and a unit extracted from that verse as input to word2vec and call this baseline S-ID.

Lardilleux and Lepage (2009) propose a simple and efficient baseline: sample-based concept induction. Words that strictly occur in the same verses are assigned to the same concept. To increase coverage, they propose to sample many different subcorpora. ${ }^{4}$ We induce concepts using this method and project them analogous to CLIQUE. We call this baseline SAMPLE.

One novel contribution of this paper is roundtrip evaluation of embeddings. We learn embeddings based on a dictionary. The question arises: are the embeddings simply reproducing the information already in the dictionary or are they improving the performance of roundtrip search?

As a baseline, we perform RTSIMPLE, a simple dictionary-based roundtrip translation method. Retrieve the pivot word $p$ in pivot language $L_{p}$ (i.e., $p \in L_{p}$ ) that is closest to the query $q \in L_{q}$. Retrieve the target unit $t \in L_{t}$ that is closest to $p$. Retrieve the pivot word $p^{\prime} \in L_{p}$ that is closest to $t$. Retrieve the unit $q^{\prime} \in L_{q}$ that is closest to $p^{\prime}$. If $q=q^{\prime}$, this is an exact hit. We run this experiment for all pivot and target languages.

Note that roundtrip evaluation tests the capability of a system to go from any language to any other language. In an embedding space, this requires two hops. In a highly multilingual dataset of $n$ languages in which not all $O\left(n^{2}\right)$ bilingual dictionaries exist, this requires four hops.

\section{Experiments and results}

\subsection{Data}

We use PBC (Mayer and Cysouw, 2014). The version we pulled on 2017-12-11 contains 1664 Bible editions in 1259 languages (based on ISO 639-3 codes) after we discarded editions that have low coverage of the New Testament. We use 7958 verses that have good coverage in these 1664 editions. The data is verse aligned; a verse of the New Testament can consist of multiple sentences. We randomly split verses $6458 / 1500$ into train/test.

\subsection{Evaluation}

For sentiment analysis, we represent a verse as the IDF-weighted sum of its embeddings. Sentiment classifiers (linear SVMs) are trained on the training set of the World English Bible edition

\footnotetext{
${ }^{4}$ We use this implementation: anymalign.limsi.fr
} 
for the two decision problems positive vs. nonpositive and negative vs. non-negative. We create a silver standard by labeling verses in English editions with the NLTK (Bird et al., 2009) sentiment classifier.

A positive vs. negative classification is not reasonable for the New Testament because a large number of verses is mixed, e.g., "Now is come salvation ... the power of his Christ: for the accuser ... cast down, which accused them before our God ..." Note that this verse also cannot be said to be neutral. Splitting the sentiment analysis into two subtasks ("contains positive sentiment: yes/no" and "contains negative sentiment: yes/no") is an effective solution for this paper.

The two trained models are then applied to the test set of all 1664 editions. All embeddings in this paper are learned on the training set only. So no test information was used for learning the embeddings.

Roundtrip translation. There are no gold standards for the genre of our corpus (the New Testament); for only a few languages out-of-domain gold standards are available. Roundtrip evaluation is an evaluation method for multilingual embeddings that can be applied if no resources are available for a language. Loosely speaking, for a query $q$ in a query language $L_{q}$ (in our case English) and a target language $L_{t}$, roundtrip translation finds the unit $w_{t}$ in $L_{t}$ that is closest to $q$ and then the English unit $w_{e}$ that is closest to $w_{t}$. If the semantics of $q$ and $w_{e}$ are identical (resp. are unrelated), this is deemed evidence for (resp. counterevidence against) the quality of the embeddings. We work on the level of Bible edition, i.e., two editions in the same language are considered different "languages".

For a query $q$, we denote the set of its $k_{I}$ nearest neighbors in the target edition $e$ by $I_{e}(q)=$ $\left\{u_{1}, u_{2}, \ldots, u_{k_{I}}\right\}$. For each intermediate entry we then consider its $k_{T}$ nearest neighbors in English. Overall we get a set $T_{e}(q)$ with $k_{I} k_{T}$ predictions for each intermediate Bible edition $e$. See Figure 5 for an example.

We evaluate the predictions $T_{e}(q)$ using two sets $G_{s}(q)$ (strict) and $G_{r}(q)$ (relaxed) of ground-truth semantic equivalences in English. Precision for a query $q$ is defined as

$$
p_{i}(q):=1 /|E| \sum_{e \in E} \min \left\{1,\left|T_{e}(q) \cap G_{i}(q)\right|\right\}
$$

where $E$ is the set of all Bible editions and $i \in$ $\{s, r\}$. We report the mean and median across a

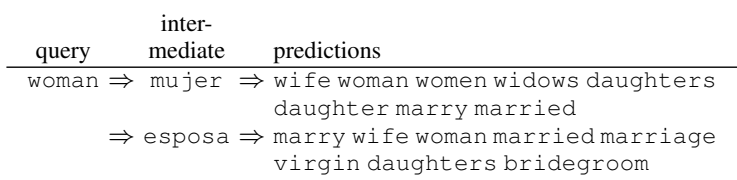

Figure 5: Roundtrip translation example for KJV and Americas Bible (Spanish). In this example $\min \left\{1,\left|T_{e}(q) \cap G_{i}(q)\right|\right\}$ equals 0 for $\mathrm{S} 1$ and R1, and 1 for S4 and S16.

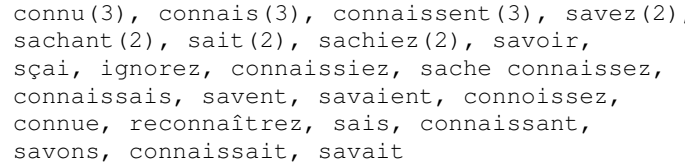

Figure 6: Intermediates aggregated over 17 French editions. $q=$ "know", $N(t)$ embeddings, S16. Intermediates are correct with two possible exceptions: "ignorez" 'you do not know'; "reconnaîtrez" 'you recognize'.

set of 70 queries selected from Swadesh (1946)'s list of 100 universal linguistic concepts.

We create $G_{s}$ and $G_{r}$ as follows. For WORD, we define $G_{s}(q)=\{q\}$ and $G_{r}(q)=L(q)$ where $L(q)$ is the set of words with the same lemma and POS as $q$. For CHAR, we need to find ngrams that correspond uniquely to the query $q$. Given a candidate ngram $g$ we consider $c_{q g}:=$ $1 / c(g) \sum_{q^{\prime} \in L(q), \operatorname{substring}\left(g, q^{\prime}\right)} c\left(q^{\prime}\right)$ where $c(x)$ is the count of character sequence $x$ across all editions in the query language. We add $g$ to $G_{i}(q)$ if $c_{q g}>\sigma_{i}$ where $\sigma_{s}=.75$ and $\sigma_{r}=.5$. We only consider queries where $G_{s}(q)$ is non-empty.

We vary the evaluation parameters $\left(i, k_{I}, k_{T}\right)$ as follows: "S1" represents $(s, 1,1)$, "S4" $(s, 2,2)$, "S16" $(s, 2,8)$, and "R1" $(r, 1,1)$.

\subsection{Corpus generation and hyperparameters}

We train with the skipgram model and set vector dimensionality to 200; word2vec default parameters are used otherwise. Each concept - the union of a set of pivot words and a set of target units linked to the pivot words - is written out as a line or (if the set is large) as a sequence of shorter lines. Training corpus size is approximately 50 GB for all experiments. We write several copies of each line (shuffling randomly to ensure lines are different) where the multiplication factor is chosen to result in an overall corpus size of approximately $50 \mathrm{~GB}$.

There are two exceptions. For BOW, we did not find a good way of reducing the corpus size, so this 


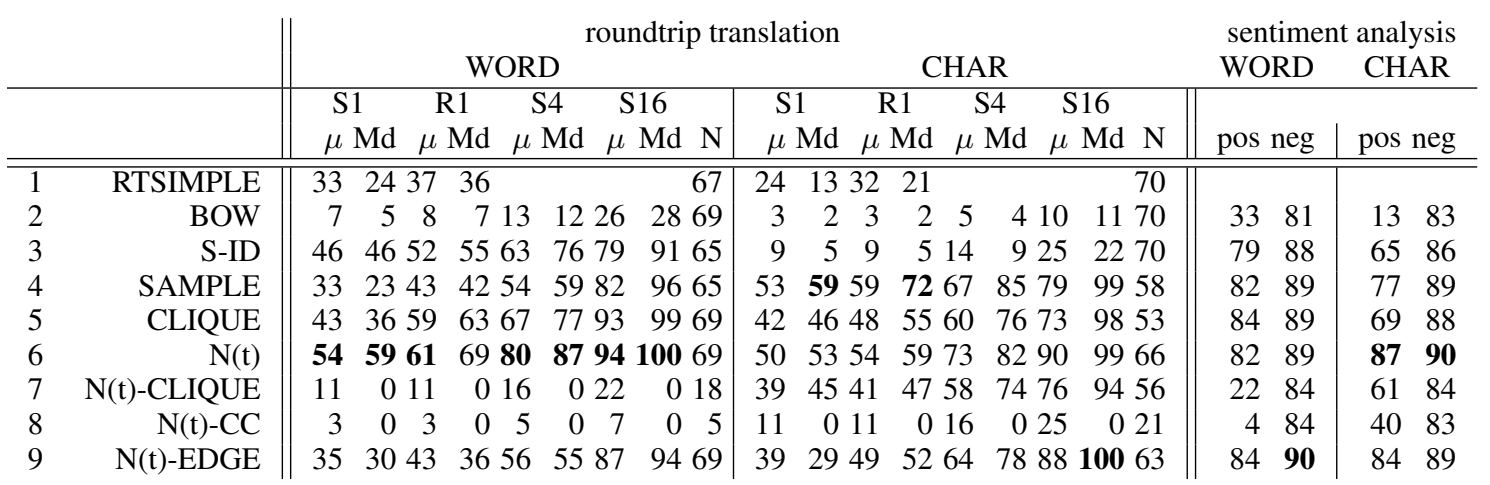

Table 3: Roundtrip translation (mean/median accuracy) and sentiment analysis $\left(F_{1}\right)$ results for wordbased (WORD) and character-based (CHAR) multilingual embeddings. $N$ (coverage): \# queries contained in the embedding space. The best result across WORD and CHAR is set in bold.

corpus is 10 times larger than the others. For SID, we use Levy et al. (2017)'s hyperparameters; in particular, we trained for 100 iterations and we wrote each verse-unit pair to the corpus only once, resulting in a corpus of about $4 \mathrm{~GB}$.

We set the $n$ parameter of $n$-grams to $n=4$ for Bible editions with $\rho<2, n=8$ for Bible editions with $2 \leq \rho<3$ and $n=12$ for Bible editions with $\rho \geq 3$ where $\rho$ is the ratio between size in bytes of the edition and median size of the 1664 editions. In $\chi^{2}$ dictionary induction, we set $\chi_{\min }=100$. In the concept induction algorithm we set $\theta=0.4$ and $\nu=0.6$. Except for SAMPLE and CLIQUE, we filter out hapax legomena.

\subsection{Results}

Table 3 presents evaluation results for roundtrip translation and sentiment analysis.

Validity of roundtrip (RT) evaluation results. RTSIMPLE (line 1) is not competitive; e.g., its accuracy is lower by almost half compared to $N(t)$. We also see that RT is an excellent differentiator of poor multilingual embeddings (e.g., BOW) vs. higher-quality ones like S-ID and $N(t)$. This indicates that RT translation can serve as an effective evaluation measure.

The concept-based multilingual embedding learning algorithms CLIQUE and $N(t)$ (lines 5-6) consistently (except S1 WORD) outperform BOW and S-ID (lines 2-3) that are not based on concepts. BOW performs poorly in our low-resource setting; this is not surprising since BOW methods rely on large datasets and are therefore expected to fail in the face of severe sparseness. S-ID performs reasonably well for WORD, but even in that case it is outperformed by $N(t)$, in some cases by a large margin, e.g., $\mu$ of 63 for S-ID vs. 80 for
$N(t)$ for S4. For CHAR, S-ID results are poor. On sentiment classification, $N(t)$ also consistently outperforms S-ID.

While S-ID provides a clearer signal to the embedding learner than BOW, it is still relatively crude to represent a word as - essentially - its binary vector of verse occurrence. Concept-based methods perform better because they can exploit the more informative dictionary graph.

Comparison of graph-theoretic definitions of concepts: $N(t)$-CLIQUE, $N(t)$-CC. $N(t)$ (line 6) has the most consistent good performance across tasks and evaluation measures. Postfiltering target neighborhoods down to cliques (line 7) and CCs (line 8) does not work. The reason is that the resulting number of concepts is too small; see, e.g., low coverages of $N=18(N(t)$-CLIQUE) and $N=5(N(t)$-CC) for WORD and $N=21$ $(N(t)$-CC) for CHAR. $N(t)$-CLIQUE results are highly increased for CHAR, but still poorer by a large margin than the best methods. We can interpret this result as an instance of a precision-recall tradeoff: presumably the quality of the concepts found by $N(t)$-CLIQUE and $N(t)$-CC is better (higher precision), but there are too few of them (low recall) to get good evaluation numbers.

Comparison of graph-theoretic definitions of concepts: CLIQUE. CLIQUE has strong performance for a subset of measures, e.g., ranks consistently second for RT (except S1 WORD) and sentiment analysis in WORD. Although CLIQUE is perhaps the most intuitive way of inducing a concept from a dictionary graph, it may suffer in relatively high-noise settings like ours.

Comparison of graph-theoretic definitions of concepts: $N(t)$ vs. $N(t)$-EDGE. Recall that $N(t)$-EDGE postfilters target neighborhoods by 


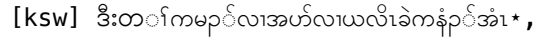

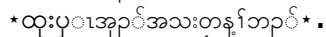

[cso] $\mathrm{Hi}^{3} \star \mathrm{Sa}^{3} \mathrm{jun}^{3} \star \mathrm{lig}^{13} \star \mathrm{ma}^{3}$ tson $^{2} \star \mathrm{tsú}^{2} \star$

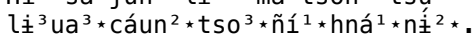

[eng] Neither $\star$ can $\star$ they $\star$ prove $\star$ the $\star$ things $*$

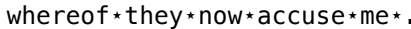

Figure 7: Verse 44024013. “*” = tokenization boundary. S'gaw Karen (ksw) is difficult to tokenize and CHAR $>$ WORD for $N(t)$. Chinanteco de Sochiapan (cso) has few types, similar to a pivot language, and CHAR $<$ WORD for $N(t)$.

\begin{tabular}{ll|ll|ll|ll}
\multicolumn{2}{c}{ N(t) } & \multicolumn{2}{c}{ S-ID } & \multicolumn{2}{c}{ SAMPLE } & \multicolumn{2}{c}{ CLIQUE } \\
\multicolumn{2}{c}{ [CHAR] } & \multicolumn{2}{c}{ [WORD] } & \multicolumn{2}{c}{ [WORD] } & \multicolumn{2}{c}{ [WORD] } \\
iso & $\Delta$ & iso & $\Delta$ & iso & $\Delta$ & iso & $\Delta$ \\
\hline arb1 & 54 & pua0 & 61 & jpn1 & 42 & mya2 & 38 \\
arz0 & 53 & sun2 & 54 & khm2 & 40 & jpn1 & 36 \\
cop3 & 49 & jpn1 & 53 & cap2 & 40 & khm3 & 34 \\
srp0 & 44 & khm3 & 53 & khm3 & 40 & bsn0 & 28 \\
cop2 & 44 & khm2 & 50 & mya2 & 39 & khm2 & 27 \\
$\ldots$ & $\ldots$ & $\ldots$ & $\ldots$ & $\ldots$ & $\ldots$ & $\ldots$ & $\ldots$ \\
pis0 & -23 & vie7 & -24 & eng8 & -7 & haw0 & -22 \\
pcm0 & -23 & kri0 & -25 & enm1 & -9 & eng4 & -23 \\
ksw0 & -24 & tdt0 & -27 & lzh2 & -9 & enm2 & -26 \\
lzh2 & -41 & eng2 & -27 & eng4 & -12 & enm1 & -26 \\
lzh1 & -51 & vie6 & -29 & lzh1 & -13 & engj & -28
\end{tabular}

Table 4: Comparison of $N(t)$ [WORD] with four other methods. Difference in mean performance (across queries) in R1 per edition. Positive number means better performance of $N(t)$ [WORD].

only considering pairs of pivot words that are linked by a dictionary edge. This "quality" filter does seem to work in some cases, e.g., best performance S16 Md for CHAR. But results for WORD are much poorer.

SAMPLE performs best for CHAR: best results in five out of eight cases. However, its coverage is low: $N=58$. This is also the reason that it does not perform well on sentiment analysis for CHAR ( $F_{1}=77$ for pos).

Target neighborhoods $N(t)$. The overall best method is $N(t)$. It is the best method more often than any other method and in the other cases, it ranks second. This result suggests that the assumption that two target units are semantically similar if they have dictionary edges with exactly the same set of pivot words is a reasonable approximation of reality. Postfiltering by putting constraints on eligible sets of pivot words (i.e., the pivot words themselves must have a certain dictionary link structure) does not consistently improve upon target neighborhoods.
WORD vs. CHAR. For roundtrip, WORD is a better representation than CHAR if we just count the bold winners: seven (WORD) vs. three (CHAR), with two ties. For sentiment, the more difficult task is pos and for this task, CHAR is better by 3 points than WORD $\left(F_{1}=87\right.$, line 6 , vs. $F_{1}=84$, lines 9/5). However, Table 4 shows that CHAR $<$ WORD for one subset of editions (exemplified by cso in Figure 7) and CHAR $>$ WORD for a different subset (exemplified by $\mathrm{ksw}$ ). So there are big differences between CHAR and WORD in both directions, depending on the language. For some languages, WORD performs a lot better, for others, CHAR performs a lot better.

We designed RT evaluation as a word-based evaluation that disfavors CHAR in some cases. The fourgram "ady@" in the World English Bible occurs in "already" (32 times), "ready" (31 times) and "lady" (9 times). Our RT evaluation thus disqualifies "ady@” as a strict match for "ready". But all 17 aligned occurrences of "ady@" are part of "ready" - all others were not aligned. So in the $\chi^{2}$ alignment interpretation, $P($ ready $\mid$ ady $@)=1.0$. In contrast to RT, we only used aligned ngrams in the sentiment evaluation. This discrepancy may explain why the best method for sentiment is a CHAR method whereas the best method for RT is a WORD method.

First NLP task evaluation on more than 1000 languages. Table 3 presents results for 1664 editions in 1259 languages. To the best of our knowledge, this is the first detailed evaluation, involving two challenging NLP tasks, that has been done on such a large number of languages. For several methods, the results are above baseline for all 1664 editions; e.g., S1 measures are above $20 \%$ for all 1664 editions for $N(t)$ on CHAR.

\section{Related Work}

Following Upadhyay et al. (2016), we group multilingual embedding methods into classes A, B, C, D.

Group A trains monolingual embedding spaces and subsequently uses a transformation to create a unified space. Mikolov et al. (2013b) find the transformation by minimizing the Euclidean distance between word pairs. Similarly, Zou et al. (2013), Xiao and Guo (2014) and Faruqui and Dyer (2014) use different data sources for identifying word pairs and creating the transformation (e.g., by CCA). Duong et al. (2017) is also simi- 
lar. These approaches need large datasets to obtain high quality monolingual embedding spaces and are thus inappropriate for a low-resource setting of 150,000 tokens per language.

Group B starts from the premise that representation of aligned sentences should be similar. Neural network approaches include (Hermann and Blunsom, 2014a) (BiCVM) and (Sarath Chandar et al., 2014) (autoencoders). Again, we have not enough data for training neural networks of this size. Søgaard et al. (2015) learn an interlingual space by using Wikipedia articles as concepts and applying inverted indexing. Levy et al. (2017) show that what we call S-ID is a strongly performing embedding learning method. We use S-ID as a baseline.

Group C combines mono- and multilingual information in the embedding learning objective. Klementiev et al. (2012) add a word-alignment based term in the objective. Luong et al. (2015) extend Mikolov et al. (2013a)'s skipgram model to a bilingual model. Gouws et al. (2015) introduce a crosslingual term in the objective, which does not rely on any word-pair or alignment information. For $n$ editions, including $O\left(n^{2}\right)$ bilingual terms in the objective function does not scale.

Group D creates pseudocorpora by merging data from multiple languages into a single corpus. One such method, due to Vulić and Moens (2015), is our baseline BOW.

Östling (2014) generates multilingual concepts using a Chinese Restaurant process, a computationally expensive method. Wang et al. (2016) base their concepts on cliques. We extend their notion of clique from the bilingual to the multilingual case. Ammar et al. (2016) use connected components. Our baseline SAMPLE, based on (Lardilleux and Lepage, 2007, 2009), samples aligned sentences from a multilingual corpus and extracts perfect alignments.

Malaviya et al. (2017), Asgari and Schütze (2017), Östling and Tiedemann (2017) and Tiedemann (2018) perform evaluation on the language level (e.g., typology prediction) for 1000+ languages or perform experiments on $1000+$ languages without evaluating each language. We present the first work that evaluates on 1000+ languages on the sentence level on a difficult task.

Somers (2005) criticizes RT evaluation on the sentence level; but see Aiken and Park (2010). We demonstrated that when used on the word/unit level, it distinguishes weak from strong embeddings and correlates well with an independent sentiment evaluation.

Any alignment algorithm can be used for dictionary induction. We only used a member of the IBM class of models (Dyer et al., 2013), but presumably we could improve results by using either higher performing albeit slower aligners or non-IBM aligners (e.g., (Och and Ney, 2003; Tiedemann, 2003; Melamed, 1997)). Other alignment algorithms include 2D linking (Kobdani et al., 2009), sampling based methods (e.g., Vulic and Moens (2012)) and EFMARAL (Östling and Tiedemann, 2016). EFMARAL is especially intriguing as it is based on IBM1 and Agic et al. (2016) find IBM2-based models to favor closely related languages more than models based on IBM1. However, the challenge is that we need to compute tens of thousands of alignments, so speed is of the essence. We ran character-based and word-based induction separately; combining them is promising future research; cf. (Heyman et al., 2017).

There is much work on embedding learning that does not require parallel corpora, e.g., (Vulić and Moens, 2012; Ammar et al., 2016). This work is more generally applicable, but a parallel corpus provides a clearer signal and is more promising (if available) for low-resource research.

\section{Summary}

We presented a new method for estimating vector space representations of words: embedding learning by concept induction. We tested this method on a highly parallel corpus and learned semantic representations of words in 1259 different languages in a single common space. Our extensive experimental evaluation on crosslingual word similarity and sentiment analysis indicates that concept-based multilingual embedding learning performs better than previous approaches.

The embedding spaces of the 1259 languages (SAMPLE, CLIQUE and $N(t)$ ) are available:

http://cistern.cis.lmu.de/comult/.

We gratefully acknowledge funding from the European Research Council (grants 740516 \& 640550) and through a Zentrum Digitalisierung.Bayern fellowship awarded to the first author. We are indebted to Michael Cysouw for making PBC available to us. 


\section{References}

Željko Agić, Anders Johannsen, Barbara Plank, Héctor Alonso Martínez, Natalie Schluter, and Anders Søgaard. 2016. Multilingual projection for parsing truly low-resource languages. Transactions of the Association for Computational Linguistics, 4.

Milam Aiken and Mina Park. 2010. The efficacy of round-trip translation for MT evaluation. Translation Journal, 14(1).

Waleed Ammar, George Mulcaire, Yulia Tsvetkov, Guillaume Lample, Chris Dyer, and Noah A Smith. 2016. Massively multilingual word embeddings. arXiv preprint arXiv:1602.01925.

Ehsaneddin Asgari and Hinrich Schütze. 2017. Past, present, future: A computational investigation of the typology of tense in 1000 languages. In Proceedings of the 2017 Conference on Empirical Methods in Natural Language Processing.

Steven Bird, Ewan Klein, and Edward Loper. 2009. Natural language processing with Python: Analyzing text with the natural language toolkit. O'Reilly Media.

Long Duong, Hiroshi Kanayama, Tengfei Ma, Steven Bird, and Trevor Cohn. 2017. Multilingual training of crosslingual word embeddings. In Proceedings of the 15th Conference of the European Chapter of the Association for Computational Linguistics.

Chris Dyer, Victor Chahuneau, and Noah A Smith. 2013. A simple, fast, and effective reparameterization of ibm model 2. In Proceedings of the 2013 Conference of the North American Chapter of the Association for Computational Linguistics: Human Language Technologies.

Manaal Faruqui and Chris Dyer. 2014. Improving vector space word representations using multilingual correlation. In Proceedings of the 14th Conference of the European Chapter of the Association for Computational Linguistics.

Dan Gillick, Cliff Brunk, Oriol Vinyals, and Amarnag Subramanya. 2016. Multilingual language processing from bytes. In Proceedings of the 2016 Conference of the North American Chapter of the Association for Computational Linguistics: Human Language Technologies.

Stephan Gouws, Yoshua Bengio, and Greg Corrado. 2015. Bilbowa: fast bilingual distributed representations without word alignments. In Proceedings of the 32nd International Conference on International Conference on Machine Learning.

Jiang Guo, Wanxiang Che, David Yarowsky, Haifeng Wang, and Ting Liu. 2016. A representation learning framework for multi-source transfer parsing. In Proceedings of the 30th AAAI Conference on Artificial Intelligence.
Karl Moritz Hermann and Phil Blunsom. 2014a. Multilingual distributed representations without word alignment. In Proceedings of the 2014 International Conference on Learning Representations.

Karl Moritz Hermann and Phil Blunsom. 2014b. Multilingual models for compositional distributed semantics. In Proceedings of the 52nd Annual Meeting of the Association for Computational Linguistics.

Geert Heyman, Ivan Vulić, and Marie-Francine Moens. 2017. Bilingual lexicon induction by learning to combine word-level and character-level representations. In Proceedings of the 15th Conference of the European Chapter of the Association for Computational Linguistics.

Alexandre Klementiev, Ivan Titov, and Binod Bhattarai. 2012. Inducing crosslingual distributed representations of words. Proceedings of the 24th International Conference on Computational Linguistics.

Hamidreza Kobdani, Alex Fraser, and Hinrich Schütze. 2009. Word alignment by thresholded twodimensional normalization. In Proceeedings of the 12th Machine Translation Summit.

Adrien Lardilleux and Yves Lepage. 2007. The contribution of the notion of hapax legomena to word alignment. In Proceedings of the 4th Language and Technology Conference.

Adrien Lardilleux and Yves Lepage. 2009. Samplingbased multilingual alignment. In Proceedings of 7th Conference on Recent Advances in Natural Language Processing.

Omer Levy, Anders Søgaard, and Yoav Goldberg. 2017. A strong baseline for learning cross-lingual word embeddings from sentence alignments. In Proceedings of the 15th Conference of the European Chapter of the Association for Computational Linguistics.

Thang Luong, Hieu Pham, and Christopher D Manning. 2015. Bilingual word representations with monolingual quality in mind. In Proceedings of the 1st Workshop on Vector Space Modeling for Natural Language Processing.

Chaitanya Malaviya, Graham Neubig, and Patrick Littell. 2017. Learning language representations for typology prediction. In Proceedings of the 2017 Conference on Empirical Methods in Natural Language Processing.

Thomas Mayer and Michael Cysouw. 2014. Creating a massively parallel bible corpus. In Proceedings of the 9th International Conference on Language Resources and Evaluation.

Ryan T. McDonald, Slav Petrov, and Keith B. Hall. 2011. Multi-source transfer of delexicalized dependency parsers. In Proceedings of the 2011 Conference on Empirical Methods in Natural Language Processing. 
I. Dan Melamed. 1997. A word-to-word model of translational equivalence. In Proceedings of the 35th Annual Meeting of the Association for Computational Linguistics and 8th Conference of the European Chapter of the Association for Computational Linguistics.

Tomas Mikolov, Kai Chen, Greg Corrado, and Jeffrey Dean. 2013a. Efficient estimation of word representations in vector space. arXiv preprint arXiv:1301.3781.

Tomas Mikolov, Quoc V Le, and Ilya Sutskever. 2013b. Exploiting similarities among languages for machine translation. arXiv preprint arXiv:1309.4168.

Franz Josef Och and Hermann Ney. 2003. A systematic comparison of various statistical alignment models. Computational Linguistics, 29(1).

Robert Östling. 2014. Bayesian word alignment for massively parallel texts. In Proceedings of the 14th Conference of the European Chapter of the Association for Computational Linguistics.

Robert Östling and Jörg Tiedemann. 2016. Efficient word alignment with Markov Chain Monte Carlo. Prague Bulletin of Mathematical Linguistics, 106.

Robert Östling and Jörg Tiedemann. 2017. Continuous multilinguality with language vectors. In Proceedings of the 15th Conference of the European Chapter of the Association for Computational Linguistics.

AP Sarath Chandar, Stanislas Lauly, Hugo Larochelle, Mitesh M. Khapra, Balaraman Ravindran, Vikas C. Raykar, and Amrita Saha. 2014. An autoencoder approach to learning bilingual word representations. In Proceedings of the 2014 Annual Conference on Neural Information Processing Systems.

Michel Simard. 1999. Text-translation alignment: Three languages are better than two. In Proceedings of the 1999 Joint SIGDAT Conference on Empirical Methods in Natural Language Processing and Very Large Corpora.

Anders Søgaard, Željko Agić, Héctor Martínez Alonso, Barbara Plank, Bernd Bohnet, and Anders Johannsen. 2015. Inverted indexing for cross-lingual nlp. In The 53rd Annual Meeting of the Association for Computational Linguistics and the 7th International Joint Conference of the Asian Federation of Natural Language Processing.

Harold Somers. 2005. Round-trip translation: What is it good for? In Proceedings of the Australasian Language Technology Workshop 2005.

Morris Swadesh. 1946. South Greenlandic (Eskimo). In Cornelius Osgood, editor, Linguistic Structures of Native America. Viking Fund Inc. (Johnson Reprint Corp.), New York.
Jörg Tiedemann. 2003. Combining clues for word alignment. In Proceedings of the 10th Conference of the European Chapter of the Association for Computational Linguistics.

Jörg Tiedemann. 2018. Emerging language spaces learned from massively multilingual corpora. arXiv preprint arXiv: 1802.00273 .

Yulia Tsvetkov, Leonid Boytsov, Anatole Gershman, Eric Nyberg, and Chris Dyer. 2014. Metaphor detection with cross-lingual model transfer. In Proceedings of the 52nd Annual Meeting of the Association for Computational Linguistics.

Shyam Upadhyay, Manaal Faruqui, Chris Dyer, and Dan Roth. 2016. Cross-lingual models of word embeddings: An empirical comparison. In Proceedings of the 54th Annual Meeting of the Association for Computational Linguistics.

Ivan Vulić and Marie-Francine Moens. 2012. Detecting highly confident word translations from comparable corpora without any prior knowledge. In Proceedings of the 13th Conference of the European Chapter of the Association for Computational Linguistics.

Ivan Vulic and Marie-Francine Moens. 2012. Subcorpora sampling with an application to bilingual lexicon extraction. In Proceedings of the 24th International Conference on Computational Linguistics.

Ivan Vulić and Marie-Francine Moens. 2015. Bilingual word embeddings from non-parallel documentaligned data applied to bilingual lexicon induction. In Proceedings of the 53rd Annual Meeting of the Association for Computational Linguistics and the 7 th International Joint Conference on Natural Language Processing, volume 2.

Rui Wang, Hai Zhao, Sabine Ploux, Bao-Liang Lu, Masao Utiyama, and Eiichiro Sumita. 2016. A novel bilingual word embedding method for lexical translation using bilingual sense clique. arXiv preprint arXiv:1607.08692.

Min Xiao and Yuhong Guo. 2014. Distributed word representation learning for cross-lingual dependency parsing. In Proceedings of the 18th Conference on Computational Natural Language Learning.

Daniel Zeman and Philip Resnik. 2008. Crosslanguage parser adaptation between related languages. In Proceedings of the 3rd International Joint Conference on Natural Language Processing.

Will Y Zou, Richard Socher, Daniel Cer, and Christopher D Manning. 2013. Bilingual word embeddings for phrase-based machine translation. In Proceedings of the 2013 Conference on Empirical Methods in Natural Language Processing. 